\title{
Common Carotid Flow Velocity is Associated with Cognition in Older Adults
}

\author{
Guo-Xiang Fu, Ya Miao, Hong Yan, Yuan Zhong
}

\begin{abstract}
Aims: To assess the relationship between carotid flow velocity and cognitive impairment in patients with mild-moderate $(<50 \%)$ carotid artery disease. Methods: We studied 407 participants with available carotid ultrasound and cognitive measures. We related peak systolic velocity (PSV) and end diastolic velocity (EDV) of internal carotid artery (ICA) and common carotid artery (CCA) and intimal medial thickness (IMT) to Mini Mental State Examination (MMSE), Clock Draw Test (CDT), Activities of Daily Living Scale (ADL)and Montreal Cognitive Assessment (MoCA). Results: EDV of CCA was significantly different in higher and lower MoCA (MMSE) groups. Multiple regression analysis demonstrated that lower EDV was significantly associated with lower MoCA (+0.459 per standard deviation (SD), p $<0.01$ for the left; +0.539 per $\mathrm{SD}, \mathrm{p}<0.01$ for the right) and CDT (odds ratio (OR) $0.093, \mathrm{p}<$ 0.05 for the left; OR) $0.120, \mathrm{p}<0.01$ for the right) scores. PSV of left CCA $(-0.205$ per SD, p $<0.05)$ and IMT $(+42.536$ per SD, p $<$ $0.001)$ were associated with ADL. PSV of right CCA was associated with MMSE $(+0.081$ per SD, p < 0.001$)$. No significant relationship between ICA flow velocity and cognitive performance was observed. Conclusions: Our preliminary data show that common carotid artery flow velocity was associated with cognitive performance.
\end{abstract}

RÉSUMÉ: La vélocité du flux dans la carotide primitive est associée à la cognition chez les adultes plus âgés. Objectifs : Le but de l'étude était de déterminer quelle est la relation entre la vélocité du flux carotidien et le déficit cognitif chez des patients atteints de maladie carotidienne de légère à modérée $(<50 \%)$. Méthode : Nous avons étudié 407 patients dont l'échographie carotidienne et l'évaluation cognitive étaient disponibles. Nous avons étudié la relation entre le pic systolique de vélocité (PSV) du flux sanguin et la vélocité en fin de diastole (VFD) au niveau de la carotide interne (CI) et de la carotide primitive (CP) ainsi que l'épaisseur intima-média (EIM) et le résultat du Mini Mental State Examination (MMSE), du test de la montre (TM), de l'échelle des activités de la vie quotidienne (AVQ) et de l'évaluation cognitive de Montréal (MoCA). Résultats : La VFD au niveau de la CP était significativement différente entre le groupe qui avait des scores plus élevés et celui qui avait des scores plus bas au MoCA (MMSE). L'analyse de régression multiple a montré qu'une VFD plus basse était associée significativement à un score plus bas au MoCA $(+0,459$ par ET, $\mathrm{p}<0,01$ pour la gauche ; + 0,539 par ET, $\mathrm{p}<0,01$ pour la droite) et au TM (RC 0,093, p <0,05 pour la gauche ; RC 0,120, p $<0,01$ pour la droite). Le PSV de la CP gauche $(-0,205$ par ET, $\mathrm{p}<0,05)$ et l'EIM $(+42,536$ par ET, $\mathrm{p}<0,001)$ étaient associés aux AVQ. Le PSV de la CP droite était associé au MMSE (+ $0,081$ par ET; $\mathrm{p}<0,001)$. Nous n'avons observé aucune relation significative entre la vélocité du flux au niveau de la CI et la performance cognitive. Conclusions : Selon nos données préliminaires, la vélocité du flux sanguin dans la carotide primitive serait associée à la performance cognitive.

Can J Neurol Sci. 2012; 39: 502-507

While stroke is a known cause of a cognitive impairment, the relationship between a carotid artery stenosis and cognitive function in individuals without a history of stroke is less clear. A number of risk factors for vascular disease are related to cognitive impairment. Hypertension, diabetes mellitus, cigarette smoking, and dyslipidemia are also associated with an increased risk of carotid artery disease ${ }^{1-6}$. Some studies have suggested that a stenosis of the internal carotid artery may be an independent risk factor for cognitive impairment ${ }^{7}$. A high-grade stenosis of the internal carotid artery may be associated with cognitive impairment even without evidence of infarction on magnetic resonance imaging ${ }^{8}$. On the other hand, it is fairly common that patients display a normal cognition despite severe carotid artery disease, highlighting the important role of an efficient collateral blood supply. Previous study found that individuals with higher grade carotid stenosis on the left (LCS) and total carotid stenosis (TCS) were less likely to demonstrate lowered cognitive performance suggesting that carotid stenosis is not detrimental to cognitive functioning 9 . The possible pathomechanisms of cognitive impairment include silent embolization and hypoperfusion. Emerging evidence showed that carotid blood flow velocities are related to patterns of resting regional cerebral blood flow in older adults, which correlated with cerebrovascular disease and cognitive activity ${ }^{10-13}$. We hypothesize that carotid artery flow velocity may relate to cognitive performance. The present study represents an attempt to correlate carotid artery flow velocity and cognitive performance in subjects with carotid artery disease.

From the Department of Gerontology, Shanghai Jiao Tong University affiliated sixth people's Hospital, Shanghai, PR China.

Received November 7, 2011. Final Revisions Submitted February 8, 2012 Correspondence to: Yuan Zhong, Department of Gerontology, Shanghai Jiao Tong University affiliated sixth people's Hospital, No. 600, Yi Shan road, Shanghai, 200233, PR China. Email: zhongyuan60@126.com 


\section{METHODS}

\section{Study Sample}

The study was initiated in 2009 as a prospective intervention study of elderly Chinese people with carotid artery disease for the purpose of determining how to prevent dementia. Approximately every month, participants visit the Department of Gerontology on Aging in Shanghai Jiao Tong University affiliated sixth people's Hospital for medical, psychological, and cognitive testing. A total of 466 participants were available for the present study. We excluded participants with carotid artery $\geq$ $50 \%$ stenosis $(n=14)$, congestive heart failure $(n=12)$, peripheral arterial disease $(n=10)$, carotid endarterectomy $(n=0)$, history of clinical stroke $(n=16)$, and other neurological diseases $(n=7)$ across all assessment visits. Following exclusions, 407 participants were available for analyses. The human ethics boards of Jiao Tong University approved the study, and subjects provided written informed consent before study participation.

\section{Carotid Ultrasound}

The Sequoia (Acuson C256, USA), combining a highresolution real-time $7.5-\mathrm{MHz} \mathrm{B}$ mode imaging system for morphological investigation and a pulsed-wave Doppler spectrum analyzer for carotid flow detection, was employed in this study. Direct visualization and Doppler flow analysis of internal and common carotid arteries was performed with longitudinal (anterioposterior, posterioanterior and lateral) and transverse views. Duplex measurements were made for peak systolic velocity (PSV), end-diastolic velocity (EDV). As carotid artery $\geq 50 \%$ stenosis defined by peak-systolic velocities $\geq 150$ $\mathrm{cm} / \mathrm{s}$ can alter hemodynamics, only patients with carotid stenosis $<50 \%$ were enrolled.

For carotid intimal medial thickness (IMT), a region $1.5 \mathrm{~cm}$ proximal to the carotid bifurcation was identified, and the IMT of the far arterial wall was evaluated as the distance between the lumen/intimal interface and the medial/adventitial interface. Specific care was taken to measure IMT in areas devoid of plaque. The IMT was measured on a frozen-frame image, magnified to achieve higher resolution of detail. The IMT measurement was obtained from five contiguous sites at approximately $1 \mathrm{~mm}$ intervals; the mean of these values was used in statistical analyses.

\section{Cognitive Measures}

The Mini Mental State Examination (MMSE) is one of the most commonly used cognitive screening measures because it is quick and easy to administer ${ }^{14,15}$. The MMSE includes specific questions related to attention, orientation, memory, calculation, visuspatial construction and language. The measure's scoring is based on 30 total points, and impairment is indicated by a score of 24 or lower. While this measure is often used to screen for memory function, it has a number of psychometric limitations, such as few executive function items. While the MMSE has good specificity (96\%), the sensitivity is poor (64\%), suggesting that cognitive changes remain undetected in a number of individuals ${ }^{16}$.

The Montreal Cognitive Assessment (MoCA) is a cognitive screening tool with high sensitivity and specificity for the detection of mild cognitive impairment ${ }^{17}$. It is a brief 30 - question test that takes around ten minutes to complete. It assesses different types of cognitive abilities, including orientation, short-term memory, executive function, language abilities, and visuospatial ability. Unlike the MMSE, the MoCA includes a clock-drawing test and a test of executive function known as Trails B. The basic Activities of Daily Living Scale $(\mathrm{ADL})^{18}$ assessed the daily life capacity results and Clock Draw Test (CDT) ${ }^{19}$ assessed executive function.

\section{Covariates}

Total cholesterol (TC), high-density lipoprotein cholesterol (HDL-C), low-density lipoprotein cholesterol (LDL-C), lipoprotein (a) (Lpa) and triglyceride (TG) were determined enzymatically (ABA-200ATC Biochromatic Analyzer; Abbott Laboratories, Irving, TX). Resting brachial systolic and diastolic blood pressure (SBP, DBP) values were obtained three times bilaterally with participants in the seated position following a five minute resting period. The SBP and DBP were defined by Korotkoff Phases I and V, respectively. Mean arterial pressure (MAP) was calculated as MAP $=(2 * \mathrm{DBP}+\mathrm{SBP}) / 3$, using the mean of the second and third blood pressure measurements.

\section{Data Analyses}

The subjects were divided into two groups, one with higher cognitive function, defined as having an MMSE score of 24 or more (MoCA score of 26 or more), and one with lower cognitive function, defined as having an MMSE score of 23 or less (MoCA score of 25 or less $)^{20,21}$. The groups were compared with respect to each factor by the Student's t-test for continuous variables or a $\chi 2$-test for categorical variables. Single regression analysis and mixed-effects regression analyses (forward stepwise) were used to determine the associations of carotid artery flow velocity and cognitive performance after adjusting for age, smoking and depressive symptoms. Age, smoking and depressive symptoms adjustment was used because they were the only significantly different variables between groups. All statistical procedures were performed with SPSS 17.0 for Windows (SPSS Inc., Chicago, IL).

\section{Results}

Baseline demographic data for the study participants are shown in Table 1. The average age in the higher MMSE (MoCA) score group was $71.6 \pm 4.6(71.2 \pm 4.3)$ and that in the lower MMSE (MoCA) score group was $72.7 \pm 5.1$ (72.9 \pm 5.0$)$. The average PSV and EDV levels in the higher MMSE score group were $74.3 \pm 20.4 \mathrm{~cm} / \mathrm{sec}$ versus $50.4 \pm 12.1 \mathrm{~cm} / \mathrm{sec}$ and that in the lower MMSE score group were $14.9 \pm 5.1 \mathrm{~cm} / \mathrm{sec}$ versus $10.5 \pm 3.3$ $\mathrm{cm} / \mathrm{sec}$, respectively. The average PSV and EDV levels in the higher MoCA score group were $75.0 \pm 21.1 \mathrm{~cm} / \mathrm{sec}$ versus $59.3 \pm 17.3 \mathrm{~cm} / \mathrm{sec}$ and that in the lower MoCA score group were $16.1 \pm 4.6 \mathrm{~cm} / \mathrm{sec}$ versus $10.2 \pm 2.5 \mathrm{~cm} / \mathrm{sec}$, respectively. Analysis by Student's t-test or $\chi 2$-test showed that age, depression and smoking habit were significantly different between the two groups of patients. Comparisons between higher and lower MMSE (MoCA) groups showed significant differences in EDV. The EDV levels were higher in the higher MMSE (MoCA) score group than the ones in the lower MMSE (MoCA) score group. The PSV was different in higher and lower MMSE groups, but 
Table 1: Analysis by Student's t-test and $\chi 2$-test between higher and lower MMSE (MoCA) groups

\begin{tabular}{|c|c|c|c|c|c|c|}
\hline Item & Higher (MMSE) & Lower (MMSE) & $p$-Value & Higher (MoCA) & Lower (MoCA) & $p$-Value \\
\hline Number & 241 & 166 & & 215 & 192 & \\
\hline Age (years) & $71.6 \pm 4.6$ & $72.7 \pm 5.1$ & $<0.001$ & $71.2 \pm 4.3$ & $72.9 \pm 5.0$ & $<0.001$ \\
\hline Gender ( $\%$ male $)$ & $45.9(111)$ & $40.0(66)$ & 0.304 & $40.9(88)$ & $42.0(81)$ & 0.404 \\
\hline Education (years) & $14.3 \pm 9.7$ & $15.1 \pm 8.8$ & 0.545 & $16.3 \pm 8.7$ & $17.1 \pm 9.8$ & 0.548 \\
\hline Smoking ( $\%$ ever) & 46.5 (112) & $31.0(51)$ & 0.022 & $48.5(104)$ & $64.4(124)$ & 0.018 \\
\hline BMI $\left(\mathrm{kg} / \mathrm{m}^{2}\right)$ & $23.8 \pm 3.5$ & $23.9 \pm 3.2$ & 0.874 & $23.7 \pm 3.3$ & $23.8 \pm 3.4$ & 0.872 \\
\hline $\mathrm{SBP}(\mathrm{mmHg})$ & $135.4 \pm 15.6$ & $133.3 \pm 19.3$ & 0.391 & $136.4 \pm 14.6$ & $134.3 \pm 18.3$ & 0.388 \\
\hline $\mathrm{DBP}(\mathrm{mmHg})$ & $74.9 \pm 9.5$ & $76.4 \pm 11.2$ & 0.288 & $75.9 \pm 8.5$ & $76.4 \pm 10.2$ & 0.298 \\
\hline LDL-c (mg/dl) & $120.9 \pm 30.6$ & $126.2 \pm 35.7$ & 0.201 & $121.9 \pm 32.6$ & $127.2 \pm 30.7$ & 0.199 \\
\hline TG $((\log )$ & $4.7 \pm 0.5$ & $4.6 \pm 0.5$ & 0.353 & $4.6 \pm 0.4$ & $4.7 \pm 0.3$ & 0.333 \\
\hline HDL-c (mg/dl)) & $56.4 \pm 18.0$ & $57.7 \pm 18.4$ & 0.567 & $55.4 \pm 18.1$ & $57.0 \pm 16.4$ & 0.665 \\
\hline Depression (\%) & 48.5 (117) & $64.4(107)$ & 0.018 & $40.4(87)$ & $25.4(49)$ & 0.017 \\
\hline Lpa $(\mathrm{mg} / \mathrm{dl}))$ & $23.1 \pm 22.9$ & $25.9 \pm 23.5$ & 0.362 & $22.1 \pm 21.9$ & $26.9 \pm 22.5$ & 0.342 \\
\hline FBG $(\mathrm{mmol} / \mathrm{l})$ & $5.1 \pm 0.3$ & $5.0 \pm 0.5$ & 0.234 & $5.0 \pm 0.3$ & $5.1 \pm 0.5$ & 0.224 \\
\hline CAD drugs use $(\%)$ & $55.2(133)$ & $62.7(104)$ & 0.261 & $65.5(141)$ & $73.2(141)$ & 0.241 \\
\hline LPSV $(\mathrm{cm} / \mathrm{sec})$ & $73.4 \pm 18.5$ & $49.8 \pm 12.7$ & 0.005 & $72.0 \pm 19.9$ & $62.7 \pm 18.6$ & 0.423 \\
\hline $\operatorname{RPSV}(\mathrm{cm} / \mathrm{sec})$ & $75.2 \pm 22.2$ & $50.9 \pm 11.5$ & 0.004 & $77.9 \pm 22.2$ & $55.8 \pm 16.0$ & 0.087 \\
\hline $\operatorname{LEDV}(\mathrm{cm} / \mathrm{sec})$ & $15.0 \pm 5.2$ & $10.1 \pm 3.8$ & 0.030 & $15.9 \pm 5.2$ & $9.8 \pm 1.9$ & 0.038 \\
\hline $\operatorname{REDV}(\mathrm{cm} / \mathrm{sec})$ & $14.7 \pm 4.9$ & $10.8 \pm 2.8$ & 0.034 & $16.2 \pm 3.9$ & $10.6 \pm 3.0$ & 0.018 \\
\hline
\end{tabular}

LPSV: peak-systolic velocity of left CCA; RPSV: peak-systolic velocity of right CCA; EDV: end diastolic velocity; CCA: common carotid artery. Lpa: Lipoprotein (a); CAD: Cardiovascular. MMSE: Mini Mental State Examination. BMI: Body Mass Index. SBP: systolic blood Pressure. DBP: diastolic blood pressure. Higher (MMSE): the group with higher MMSE scores (24 or more), Lower (MMSE): the group with lower MMSE scores (23 or less). Higher (MoCA): the group with higher MMSE scores (26 or more), Lower (MoCA): the group with lower MoCA scores (25 or less).

did not reach statistical significance in the higher and lower MoCA score group (Table 1).

The PSV and EDV of bilateral common carotid artery (CCA) were both related to MMSE and CDT. Participants with lower PSV and EDV level had a lower MMSE and CDT score. Lower EDV level of bilateral CCA was also related to lower MoCA score $(\mathrm{p}<0.01)$. Lipoprotein (a) (Lpa) was inversely related to
ADL and CDT. (Table 2). When using the full multivariable adjusted model, lower EDV of bilateral CCA remained significant with lower MoCA $(+0.459$ per $\mathrm{SD}, \mathrm{p}<0.01$ for the left; +0.539 per SD, $p<0.01$ for the right ) and CDT (OR 0.093, $\mathrm{p}<0.05$ for the left; OR $0.120, \mathrm{p}<0.01$ for the right) score. We observed that CCA IMT was significantly associated with lower ADL score (42.536 per SD \pm SE 6.602, $\mathrm{p}<0.001)$. The PSV of

Table 2: Relationship between CCA flow velocity, atherosclerosis measures and cognitive performance

\begin{tabular}{|c|c|c|c|c|c|c|}
\hline \multirow[t]{2}{*}{ CM factors } & \multicolumn{2}{|c|}{$\operatorname{PSV}(\mathrm{cm} / \mathrm{sec})$} & \multicolumn{2}{|c|}{$\operatorname{EDV}(\mathrm{cm} / \mathrm{sec})$} & \multirow[t]{2}{*}{ IMT (mm) } & \multirow[t]{2}{*}{$\mathrm{Lpa}(\mathrm{mmol} / \mathrm{l})$} \\
\hline & Left & Right & Left & Right & & \\
\hline MMSE & $0.062(0.026)^{*}$ & $0.081(0.019)^{\xi}$ & $0.237(0.099)^{*}$ & $0.265(0.111)^{*}$ & $-4.71(3.266)$ & $-0.089(0.053)$ \\
\hline MoCA & $0.097(0.054)$ & $0.129(0.039){ }^{\#}$ & $0.533(0.172)^{\#}$ & $0.633(0.213)^{\#}$ & $-4.945(3.847)$ & $-0.09(0.073)$ \\
\hline ADL & $-0.384(0.134)^{\#}$ & $-0.12(0.141)$ & $-1.199(0.543)^{*}$ & $-0.356(0.703)$ & $32.043(1.836)^{\xi}$ & $-0.32(0.15)^{*}$ \\
\hline CDT & $0.032(0.015){ }^{*}$ & $0.033(0.012)^{*}$ & $0.141(0.053)^{*}$ & $0.172(0.057)^{\#}$ & $-1.075(0.854)$ & $-0.038(0.013)^{\#}$ \\
\hline
\end{tabular}

PSV: peak-systolic velocities; EDV: end diastolic velocity velocities; IMT: Intima-media thickness. Lpa: Lipoprotein a MoCA: Montreal Cognitive Assessment; ADL: Activities of Daily Living Scale; CDT: Clock Draw Test. MMSE: Mini Mental State Examination. CM: cognitive measures $* \mathrm{p}<0.05 \quad \# \mathrm{p} \leq 0.01 \quad \xi \mathrm{p}<0.001$. Effects on MMSE, MoCA and ADL are Standardized Beta correlation coefficient (Standard Error). Effect on CDT is Odds Ratio (Standard Error). 
Table 3: Mixed-Effects Regression Models predicting cognitive performance from CCA flow velocity and atherosclerosis measures

\begin{tabular}{|c|c|c|c|c|c|}
\hline \multirow[t]{2}{*}{$\mathrm{CM}$ factors } & \multicolumn{2}{|c|}{ PSV $(\mathrm{cm} / \mathrm{sec})$} & \multicolumn{2}{|c|}{$\operatorname{EDV}(\mathrm{cm} / \mathrm{sec})$} & \multirow[t]{2}{*}{ IMT (mm) } \\
\hline & Left & Right & Left & Right & \\
\hline MMSE & NS & $0.081(0.019)^{\xi}$ & NS & NS & NS \\
\hline $\mathrm{MoCA}$ & NS & NS & $0.459(0.137)^{\#}$ & $0.539(0.166)^{\#}$ & NS \\
\hline ADL & $-0.205(0.097) *$ & NS & NS & NS & $42.536(6.602)^{\xi}$ \\
\hline CDT & NS & NS & $0.093(0.041)^{*}$ & $0.120(0.042)^{\#}$ & NS \\
\hline
\end{tabular}

PSV: peak-systolic velocities; EDV: end diastolic velocity velocities; IMT: intima-media thickness; MoCA: Montreal Cognitive Assessment; MMSE: Mini Mental State Examination; ADL: Activities of Daily Living Scale; CCA: common carotid artery; CDT: Clock Draw Test.; CM: cognitive measures. ${ }^{*} \mathrm{p}<0.05$, \# $\mathrm{p} \leq 0.01, \xi \mathrm{p}<0.001$. Effects on MMSE, MoCA and ADL are Standardized Beta correlation coefficient (Standard Error). Effect on CDT is Odds Ratio (Standard Error).

right CCA was significantly associated with MMSE $(0.081$ per $\mathrm{SD} \pm \mathrm{SE0} .019, \mathrm{p}<0.001)$, while PSV of left CCA was inversely related to ADL (-0.205 per SD \pm SE 0.097, p<0.05) (Table 3).

Unlike for common carotid artery flow velocity, no significant relationship between internal carotid artery flow velocity and cognitive performance was observed (data not shown).

\section{DISCUSSION}

Recent population-based studies have suggested that asymptomatic carotid atherosclerosis is related to poorer neuropsychological performance even with mild degrees of stenosis ${ }^{1,2}$

The measurement of carotid diameter and flow velocity may be inaccurate in patients with severe carotid atherosclerosis when a stenosis alters blood volume. In our study, carotid artery $\geq$ $50 \%$ stenosis participants were excluded. We found that lower EDV of bilateral CCA was significantly associated with lower MoCA scores and CDT scores.

Some studies have demonstrated that CCA shear stress decreased with age, blood pressure, and body mass index and was associated with CCA intima-media thickness independently of other hemodynamic, clinical, or biochemical factors ${ }^{22}$. Few studies have focused on the relationship between Doppler parameters and cognitive performance. Urabe et al pointed out that the EDV of CCA was markedly lower and resistance index was significantly higher in patients with embolic middle cerebral artery (MCA) infarcts than in patients with thrombotic MCA infarct $^{23}$.

Bai et al's study showed that lower blood flow velocity is associated with ischemic stroke independently of carotid atherosclerosis and cardiovascular risk factors ${ }^{24}$. End-diastolic velocity increase predicts recanalization and neurological improvement in patients with ischemic stroke with proximal arterial occlusions receiving reperfusion therapies, which was associated with cognitive impairment ${ }^{25}$. Another study reported that EDV below $16 \mathrm{~cm} / \mathrm{sec}$ was a significant single predictor of ischemic stroke and recurrent ischemic stroke events ${ }^{26}$.

Several studies reported ethnic differences in the location of lesions in cerebrovascular occlusive disease $\mathrm{e}^{27-29}$ and in large artery stiffening ${ }^{30}$. Asian stroke patients are more likely to have higher prevalence of intracranial vessel stenosis than extracranial carotid disease ${ }^{31}$. In our study, the EDV was below $16 \mathrm{~cm} / \mathrm{sec}$, suggesting our population is more likely to have higher prevalence of intracranial vessel stenosis and ischemic stroke.

A lower EDV and higher RI may be considered signs of aging ${ }^{12,32-34}$. Vascular aging is thought to be associated with structural and functional alterations, including intima-media thickening, arterial enlargement, and deterioration of elastic wall properties with vascular stiffening ${ }^{35}$. Our findings, however, cannot be solely explained by age, because we adjusted for age in our analyses.

A previous study reported that the measures of right internal carotid artery (ICA) flow volume and MMSE scores were significantly lower in patients with dementia, suggesting the right ICA flow volume may be related to MMSE scores ${ }^{36}$. Similar to the results, we found that PSV of right CCA was significantly associated with MMSE, while PSV of left CCA was inversely related to ADL. This difference may be due to the different predominance of testing and cognitive domains. MMSE, driven by language (a left-sided brain function), would be more affected by PSV of right CCA, while ADL driven by executive function (a right-sided brain function), would be more affected by PSV of left CCA.

Intimal medial thickness has been considered a subclinical marker of early atherosclerosis. Our data is supportive of findings in previous studies such as the $\mathrm{CHS}^{2}$, which found increased CCA IMT was associated with lower cognitive performance, but no difference was found between the left and right sides. Similar to the results in the CHS cohort, we found that CCA IMT was significantly associated with lower activities of daily living scale score. We did not observe a different relation between left and right-sided measures and cognitive performance.

Atherothrombotic stenosis originating in the ICA is a common cause of ischemic stroke in the carotid territory, and the risk of stroke increases with the severity of carotid stenosis ${ }^{37}$. On the other hand, it is also well recognized that the simple presence of carotid stenosis does not predict the presence or degree of 
hemodynamic compromise in the distal cerebral circulation ${ }^{38,39}$. Blood flow via poststenotic ICA and intracranial collaterals determines cerebral blood flow in these patients.

Poststenotic ICA flow measured with transoral carotid ultrasonography reflects collateral flow and cerebral hemodynamics in patients with severe carotid stenosis ${ }^{40}$. In our study, no significant relationship between internal carotid artery flow velocity and cognitive performance was observed. That may be due to the exclusion of high-grade carotid stenosis $(>$ $50 \%$ ) patients

Some limitations should be considered in the present case. First, the present study was performed with cross-sectional design. The patients are being followed longitudinally, and a follow-up analysis will be reported in the future. Second, the study recruited patients with relatively mild carotid stenosis status. Individuals who had history of clinical stroke, angioplasty or high-grade carotid stenosis ( $\geq 50 \%$ ) were not accepted into the study, and this group of older patients therefore did not represent the general population with respect to their vascular health. Third, the present study did not include brain imaging. We excluded only the participants with history of clinical stroke. An analysis of subjects who underwent brain magnetic resonance imaging (MRI) was recently reported elsewhere and revealed that cognitive decline in carotid artery atherosclerosis was associated with silent cerebral infarcts $(\mathrm{SCI})^{41}$. However, the relationship between the present results and the results of MRI analysis requires further investigation. Variables mediating or moderating the relation between carotid flow velocity and accelerated cognitive decline also necessitate further examination.

\section{Conclusion}

We found that lower blood flow velocity (particularly EDV) of common carotid artery was significantly associated with lower cognitive performance. The precise underlying mechanism explaining the relationship between carotid atherosclerosis and cognitive performance in our study is unclear, but our data suggest that common carotid flow velocity may be a better marker for cognitive impairment than carotid atherosclerosis markers such as CCA-IMT. Therefore, we believe that these data could prove to be useful in the evaluation of cognitive decline in patients with carotid artery disease. Future research should continue to examine which cognitive domains are particularly related to carotid flow velocity and other sub-clinical vascular disease measures.

\section{ACKNOWLEDGMENTS}

The authors thank the Neuropsychological Test clinical core staff of the Gerontology Department for their assistance. This research was supported by the Research Program of Shanghai Jiao Tong University affiliated sixth people's Hospital and No.1302.

\section{REFERENCES}

1. Zhong W, Cruickshanks KJ, Huang GH, et al. Carotid atherosclerosis and cognitive function in midlife: the beaver dam offspring study. Atherosclerosis. 2011;219(1):330-3.

2. Arntzen KA, Mathiesen EB. Subclinical carotid atherosclerosis and cognitive function. Acta Neurol Scand Suppl. 2011;(191):18-22.
3. Silvestrini M, Viticchi G, Falsetti L, et al. The role of carotid atherosclerosis in Alzheimer's disease progression. J Alzheimers Dis. 2011;25(4):719-26.

4. Sander K, Bickel H, Förstl H et al. Carotid- intima media thickness is independently associated with cognitive decline. The INVADE study. Int J Geriatr Psychiatry. 2010;25(4):389-94.

5. Rafnsson S, Deary IJ, Whiteman MC, Rumley A, Lowe GD, Fowkes FG. Haemorheological predictors of cognitive decline: the Edinburgh Artery Study. Age Ageing. 2010;39(2):217-22.

6. Romero JR, Beiser A, Seshadri S, et al. Carotid artery atherosclerosis, MRI indices of brain ischemia and aging and cognitive impairment: The Framingham Study. Stroke. 2009;40 (5): 1590-6.

7. Martinić-Popović I, Lovrencić-Huzjan A, Demarin V. Assessment of subtle cognitive impairment in stroke-free patients with carotid disease. Acta Clin Croat. 2009;48(3):231-40.

8. Johnston SC, O'Meara ES, Manolio TA, et al. Cognitive impairment and decline are associated with carotid artery disease in patients without clinically evident cerebrovascular disease. Ann Intern Med. 2004;140(4):237-47.

9. Fergenbaum JHS, Bruce SW, Lou AJG, Hanley C, Greenwood TK. Young carotid atherosclerosis and a reduced likelihood for lowered cognitive performance in a Canadian First Nations population. Neuroepidemiology. 2009;33:321-8.

10. Sojkova J, Najjar SS, Beason-Held LL, et al. Intima-media thickness and regional cerebral blood flow in older adults. Stroke. 2010;41(2):273-9.

11. Kitaoka K, Miura H, Kitamura M, Akutagawa M, Kinouchi Y, Yoshizaki K. Feed-forward changes in carotid blood flow velocity during active standing. Neurosci Lett. 2011;487(2): 240-5.

12. Takeuchi S, Karino T. Flow patterns and distributions of fluid velocity and wall shear stress in the human internal carotid and middle cerebral arteries. World Neurosurg. 2010;73(3):174-85.

13. Fukuhara T, Hida K, Manabe Y, et al. Reduced flow velocity in the internal carotid artery independently of cardiac hemodynamics in patients with cerebral ischemia. J Clin Ultrasound. 2007;35 (6):314-21.

14. Folstein MF, Folstein SE, McHugh PR. "Mini-mental state:" a practical method for grading the cognitive state of patients for the clinician. J Psychiatr Res. 1975;12:189-98.

15. Malloy PF, Cummings JL, Coffey CE, et al. Cognitive screening instruments in neuropsychiatry: a report of the Committee on Research of the American Neuropsychiatric Association. J Neuropsychiatry Clin Neurosci. 1997;9:189-97.

16. Lomholt RK, Jurgensen KS. The minimental state examination in screening of cognitive dysfunction and dementia. Ugeskr Laeger. 1998;160:7251-4.

17. Nasreddine ZS, Phillips NA, Bédirian V, et al. The Montreal cognitive assessment, moca: a brief screening tool for mild cognitive impairment. Am Geriatr Soc. 2005;53(4):695-9.

18. Royall DR, Cordes JA, Polk M. CLOX: an executive drawing task. J Neurol Neurosurg Psychiatry. 1998;64:588-94.

19. Chodosh J, Miller-Martinez D, Aneshensel CS, Wight RG, Karlamangla AS. Depressive symptoms, chronic diseases, and physical disabilities as predictors of cognitive functioning trajectories in older Americans. J Am Geriatr Soc. 2010;58(12): 2350-7.

20. Tombaugh TN, McIntyre NJ. The mini-mental state examination: a comprehensive review. J Am Geriatr Soc. 1992;40:922-35.

21. Damian AM, Jacobson SA, Hentz JG, et al. The Montreal cognitive assessment and the mini-mental state examination as screening instruments for cognitive impairment: item analyses and threshold scores. Dement Geriatr Cogn Disord. 2011;31(2): 126-31.

22. Carallo C, Irace C, Pujia A, et al. Evaluation of common carotid hemodynamic forces. Relations with wall thickening. Hypertension. 1999;34:217-21.

23. Urabe T, Shioya-Morikawa N. Differentiation of embolic and thrombotic middle cerebral artery occlusion using ultrasonic carotid flow velocity analysis. J Neurol Sci . 1995;128:181-7.

24. Bai CH, Chen JR, Chiu HC, Pan WH. Lower blood flow velocity, higher resistance index, and larger diameter of extracranial 
carotid arteries are associated with ischemic stroke independently of carotid atherosclerosis and cardiovascular risk factors. J Clin Ultrasound. 2007;35(6):322-30.

25. Alexandrov AV, Tsivgoulis G, Rubiera M, et al. End-diastolic velocity increase predicts recanalization and neurological improvement in patients with ischemic stroke with proximal arterial occlusions receiving reperfusion therapies. Stroke. 2010; 41(5):948-52.

26. Dilic M, Kulic M, Balic S, et al. Cerebrovascular events: correlation with plaque type, velocity parameters and multiple risk factors. Med Arh. 2010;64(4):204-7.

27. Feldmann E, Daneault N, Kwan E, et al. Chinese-white differences in the distribution of occlusive cerebrovascular disease. Neurology. 1990;40:1541-5.

28. Suh DC, Lee SH, Kim KR, et al. Pattern of atherosclerotic carotid stenosis in Korean patients with stroke: different involvement of intracranial versus extracranial vessels. AJNR Am J Neuroradiol. 2003;24:239-44.

29. Liu HM, Tu YK, Yip PK, et al. Evaluation of intracranial and extracranial carotid steno-occlusive diseases in Taiwan Chinese patients with MR angiography: preliminary experience. Stroke. 1996;27:650-3

30. Din-Dzietham R, Couper D, Evans G, et al. Arterial stiffness is greater in African Americans than in whites: evidence from the Forsyth County, North Carolina, ARIC cohort. Am J Hypertens. 2004;17:304-13.

31. Tan TY, Chang KC, Liou CW, et al. Prevalence of carotid artery stenosis in Taiwanese patients with one ischemic stroke. J Clin Ultrasound. 2005;33:1-4.

32. Bes A, Guell A, Braak L, et al. Influence of age on several parameters of the Doppler signal at the carotid level [in French]. Rev Electroencephalogr Neurophysiol Clin. 1980;10:190-6.

33. Schoning M, Walter J, Scheel P. Estimation of cerebral blood flow through color duplex sonography of the carotid and vertebral arteries in healthy adults. Stroke. 1994;25:17-22.
34. Schmidt-Trucksass A, Grathwohl D, Schmid A, et al. Structural, functional, and hemodynamic changes of the common carotid artery with age in male subjects. Arterioscler Thromb Vasc Biol. 1999;(19):1091-7.

35. Dijk JM, Algra A, van der Graaf Y, et al. Carotid stiffness and the risk of new vascular events in patients with manifest cardiovascular disease. The SMART study. Eur Heart J. 2005; 26:1213-20.

36. Albayrak R, Yaman M, Uslan I, Degirmenci B, Acar M, Haktanir A. Evaluation of total cerebral blood flow volume in cerebral atrophy. Neurosci Lett. 2006;15;399(1-2):124-8.

37. Henderson RD, Eliasziw M, Fox AJ, Rothwell PM, Barnett HJ. Angiographically defined collateral circulation and risk of stroke in patients with severe carotid artery stenosis: North American Symptomatic Carotid Endarterectomy Trial (NASCET) group. Stroke. 2000;31:128-32.

38. Powers WJ, Press GA, Grubb RL, Gado M, Raichle ME. The effect of hemodynamically significant carotid artery disease on the hemodynamic status of the cerebral circulation. Ann Intern Med. 1987; 106:27-34

39. Powers WJ. Cerebral hemodynamics in ischemic cerebrovascular disease. Ann Neurol. 1991;29:231-40.

40. Kamouchi M, Kishikawa K, Okada Y, Inoue T, Ibayashi S, Iida M. Poststenotic flow and intracranial hemodynamics in patients with carotid stenosis: transoral carotid ultrasonography study. Am J Neuroradiol. 2005;26:76-81.

41. Vermeer SE, Prins ND, den Heijer T, Hofman A, Koudstaal PJ, Breteler MM. Silent brain infarcts and the risk of dementia and cognitive decline. N Engl J Med. 2003;27;348(13):1215-22. 